\title{
Desarrollo de la investigación contable en el Centro Colombiano de Investigación Contable*
}

Claudia Barrios Álvarez Profesora-investigadora, Pontificia Universidad Javeriana Cali. claudiabarrios@javerianacali.edu.co

\section{Tatiana Fúquene}

Sánchez.

Investigadora, tatiana.fuquene@saincsa.com

\section{Jorge Eduardo Lemos}

de la Cruz

Profesor-investigador, Universidad Libre de Colombia. jeduardo@univalle.edu.co

* Este artículo se inscribe en el proyecto "Utilidad de la información contable en Colombia en: los modelos de reporting, prácticas investigativas y prácticas contables", adscrito al grupo de investigación Pensamiento y Praxis Contable de la Pontificia Universidad Javeriana Cali.

\section{Resumen}

En este trabajo se hace una revisión de los resultados de la investigación que se ha llevado a cabo en veintitrés años de trabajo en el Centro Colombiano de Investigación Contable (Ccinco). Este centro reúne a profesores universitarios (contadores públicos) vinculados con distintas universidades de Colombia que efectúan y promueven la investigación contable. Esta organización surgió en 1987 en un contexto en el cual la investigación contable empezó a ser reconocida en Colombia como una actividad fundamental para el desarrollo de la disciplina y la profesión. Este trabajo utiliza la propuesta taxonómica de Jorge Túa Pereda sobre los enfoques de investigación contable como herramienta para la identificación y clasificación de las hipótesis y problemas escogidos por los investigadores, y sobre éstos sondear el análisis epistemológico de su formulación, así como también las pruebas de contrastación de las hipótesis encontradas.

Palabras clave: investigación contable, contabilidad, Centro Colombiano de Investigación Contable (Ccinco), enfoques de investigación contable. 


\title{
Development of the accounting research in the Colombian Center for Resear- ch on Accounting. Research approach
}

\begin{abstract}
The present work is a synthesis and analysis which reviews the research done by Ccinco (Centro Colombiano de Investigación Contable or the Colombian Center for Research on Accounting) for the last 23 years. This research center brings together Public Accounting professors from various universities in Colombia with the objective of developing and promoting accounting research. This organization was started in 1987 in a context when accounting research was being recognized in Colombia as a key activity in the development of both the accounting discipline and the accounting profession. Jorge Túa Pereda's taxonomic proposal for an approach to accounting research is used here as a tool for the identification and classification of the hypotheses and problems chosen by researchers, and also explored is the epistemological analysis of their formulation which leads to scrutiny and discussion of their associated regulation.
\end{abstract}

Keywords: Accounting Research, Accounting, Colombian Center for Research on Accounting and approach to accounting research.

\section{Introducción}

El Centro Colombiano de Investigación Contable (Ccinco) es una organización que congrega contadores públicos, quienes son profesores vinculados a distintas universidades de Colombia: Édgar Gracia López, actual director del Centro, Jack Alberto Araujo, Rafael Franco Ruiz, Hernán Quintero Castro, Efrén Danilo Ariza, Harold Álvarez, William Rojas Rojas, Olver Quijano Valencia y Guillermo León Martínez. También estuvo vinculado a este Centro Jorge Enrique Burbano.

El Ccinco, que surge en el año 1987, establece en sus estatutos de constitución las siguientes áreas de conocimiento: teoría contable, metodología de la investigación contable, epistemología y contabilidad, teoría del control, teoría fiscal, educación contable, relaciones de la contabilidad con otras áreas del conocimiento, reglamentación profesional, historia de la contabilidad, la contaduría y la educación contable, relaciones contabilidad-contaduría-sociedad, relación entre micro y macrocontametría, planeación y presupuestos, contabilidad y desarrollo económico, contabilidad nacional, contabilidad y ciencia política, moral profesional y contabilidad, control e informática (Ccinco, 1987). 
El desarrollo de la investigación contable en el Ccinco es estudiado a partir de la identificación de los problemas e hipótesis escogidas por los miembros del Centro en sus publicaciones de los últimos veintitrés años.

Estas hipótesis son clasificadas de acuerdo con la taxonomía propuesta por Túa Pereda, la cual es resultado de la exploración histórica sobre el desarrollo de la investigación contable en el mundo. Esta taxonomía ofrece un referente comparativo para identificar las hipótesis que se sostienen desde su criterio epistemológico y cuáles de ellas, descriptivamente, se acercan a este estatus, y si existen contrastaciones empíricas que las sustenten o argumentos que lógica y analíticamente las soporten.

La elección de la metodología propuesta por Jorge Túa Pereda está justificada en la clasificación de los enfoques de investigación, a través de la revisión de los objetivos, hipótesis y problemas de investigación que configuran cada enfoque. La revisión histórica y epistemológica que efectúa este autor permite que se comparen con las hipótesis y problemas que los investigadores del Ccinco han proferido desde los distintos intereses extra e intrateóricos.

En primer lugar, este trabajo de investigación analiza el contexto histórico social en el cual se desarrolla la investigación contable del Ccinco; la ubicación histórica, a manera de sinopsis, que establece un marco general para la reflexión de posibles lugares interpretativos en las hipótesis formuladas y problemas planteados por los investigadores. El plano histórico no se estudia en este artículo, pero sí en la ruta metodológica elegida por los autores para sustentar el desarrollo de la investigación.

En segundo lugar, y de manera sistemática, se revisan y clasifican los artículos, capítulos de libros y libros (de un total de 192 artículos, 6 capítulos de libros y 20 libros) de la producción del Ccinco en veintitres años de trabajo. Las temáticas que predominan en las publicaciones revisadas son: contabilidad social, ambiental, educación contable, historia de la contabilidad y la contaduría pública, normalización, regulación, control, revisoría fiscal, teoría, metodología y epistemología de la contabilidad.

De manera particular, para acceder a las publicaciones de los miembros del centro de investigación fueron visitadas bibliotecas privadas y personales, en distintas 
ciudades del país, lo cual motivó también la aplicación de entrevistas y recolección de relatos sobre la investigación realizada en dicho centro ${ }^{1}$.

Una vez situados en el aspecto histórico y procesada la información recolectada, en la tercera etapa se identifican las principales hipótesis y problemas trabajados por los distintos autores del Ccinco para verificarlos con la propuesta taxonómica de Jorge Túa Pereda, y sobre éstos sondear el análisis epistemológico de su formulación, así como también las pruebas de contrastación de las hipótesis encontradas. Esta etapa es la que se analiza en el presente artículo.

\section{Propuesta taxonómica de Jorge Túa Pereda}

El contenido de los estados financieros fue severamente cuestionado en la década del sesenta del siglo pasado, lo que ocasionó que se desarrollará el paradigma de la utilidad, el cual surgió por la crisis de 1929, dado el colapso presentado en la bolsa de valores no previsto a partir de la información contable financiera. Este episodio desencadenó fuertes cuestionamientos sobre cómo era trabajada la información contable, planteando la necesidad de orientar sus objetivos de información hacia los usuarios, ya no sólo a los gerentes y acreedores desde el paradigma del cálculo del beneficio, sino también a los inversionistas y accionistas, concibiendo que el uso de la información fuera más social.

A partir de este momento se consideró fundamental la relevancia de la información contable con relación a la satisfacción de las necesidades de sus usuarios, cuestionándose sobre cuáles son los usuarios, a qué deben responder los objetivos de la información contable y su dimensión. Según Túa (1989) las distintas formas de abordar estas cuestiones han dado lugar a la configuración de diversos enfoques contables, respondiendo a la pregunta de cómo satisfacer las necesidades de los usuarios de dicha información.

\footnotetext{
${ }^{1}$ La búsqueda bibliográfica de la producción de los miembros del Ccinco se inicia en 1998 y culmina en el 2002. En este tiempo se recogen los trabajos de los diez miembros del centro de investigación vinculados hasta ese momento, incluyendo sus primeras publicaciones en el año 1979 hasta el 2002. La inclusión de las publicaciones se delimita hasta el momento en que se inicia la sistematización de los trabajos — año 2002—, después de esta fecha se han vinculado nuevos miembros del Ccinco y se han desarrollado nuevas actividades y publicaciones, sin embargo para la viabilidad metodológica de esta investigación se delimita este momento para concluir la búsqueda de datos y fuentes primarias.
} 
Un análisis de los enfoques en la contabilidad refleja la evolución del pensamiento contable, pues implica ofrecer una nueva configuración, así como identificar las concepciones de la contabilidad, el método contable, sus aplicaciones y las teorías, hipótesis e instrumentos de investigación compartidos. Estos elementos básicos son retomados por Túa a partir de los aportes de Belkaoui (1993), quien a su vez los retoma de los enfoques planteados por la American Accounting Association (AAA) en su publicación Statement on Accounting Theory and Theory Acceptance AAA (1977 citado en Belkaoui, 1993) estructurándolos desde una postura socioepistemológica e incorporando los elementos de Kuhn para su análisis.

Los enfoques de investigación trabajados por Túa Pereda son: el inductivo positivista, el deductivo normativo, el de los modelos de decisión y la capacidad predictiva, el del comportamiento agregado del mercado, el del inversionista individual, el del valor económico de la información, el neopositivismo inductivo y el de la teoría de los hechos. Túa presenta los enfoques de investigación señalando en cada uno sus principales características, las aportaciones más representativas y la investigación empírica desarrollada bajo cada enfoque ${ }^{2}$. Para este artículo se eligió la propuesta taxonómica de Túa por llevar a cabo la identificación de las hipótesis de trabajo de los autores en sus textos como rasgo característico para la clasificación en los enfoques.

La producción académica de los miembros del Ccinco es clasificada en los enfoques de investigación utilizando un método analítico, cuyos parámetros de selección se definen por la observación de rasgos comunes dentro de los trabajos y la identificación de las hipótesis de trabajo formuladas por los miembros del Centro.

Para analizar el desarrollo de la investigación contable del Ccinco es necesario delimitar el trabajo del campo disciplinar y profesional para posteriormente identificar las hipotésis planteadas en la producción académica de sus miembros como un criterio para clasificarla en los enfoques contenidos en esta propuesta.

\footnotetext{
${ }^{2}$ Para ampliar más información sobre la exploración histórica sobre el desarrollo de la investigación contable en el mundo realizado por Túa Pereda y la configuración de los enfoques de investigación, veáse TÚA, P. J. (1989). La investigación empírica en contabilidad. La hipótesis de eficiencia". Madrid: Instituto de Contabilidad y Auditoría de Cuentas/ Ministerio de Economía y Hacienda.
} 


\section{Enfoques de investigación en Ccinco}

Los trabajos del ámbito profesional publicados por los miembros del Ccinco, aunque condicionan y proporcionan datos sobre el desarrollo de la investigación contable del Centro, no clasifican en los enfoques escogidos en esta investigación, dado que el eje principal de la taxonomía propuesta por Túa son los trabajos del ámbito disciplinar tanto a nivel teórico como técnico.

Es importante señalar que la contabilidad es la disciplina que guía la práctica contable, entendida a partir de un nivel metateórico, teórico y técnico, así como el campo de acción del contador. La definición de contabilidad no es universal, algunos autores la han definido como arte, técnica, disciplina o ciencia social, siendo múltiples las hipótesis sobre la definición del campo de la realidad que explica o predice.

\section{Textos disciplinares y profesionales}

Los trabajos disciplinares son aquellos que desarrollan investigación básica o empírica, cuyo objetivo es abordar problemas contables a nivel técnico, teórico y epistemológico, lo cual se refleja en la construcción del cuerpo teórico que fundamenta las prácticas contables (técnicas) haciendo uso de conceptos o metodologías de otras disciplinas.

Por su parte, los trabajos profesionales estudian desde posturas críticas los problemas o situaciones del ejercicio profesional en el que se resuelven problemas de la técnica que son particulares de cada contexto; es decir, no son generalizables a todo el saber contable, sino a su aplicación. En el caso de la contaduría, se encuentra guiado por la ética del contador y las aplicaciones de la contabilidad como la auditoría, revisoría fiscal y la regulación de la práctica contable.

Para determinar el campo en que es desarrollada una investigación contable es necesario tener en cuenta el tema abordado para definir si las temáticas son profesionales o disciplinares; las primeras están relacionadas con el ejercicio profesional y las segundas estudian, a nivel propositivo o crítico, conceptos estructurales del saber de la contabilidad. En segundo término, por el tipo de argumentación, los trabajos pueden elaborarse desde argumentos científicos, económicos, psicológicos, filosóficos y/o sociológicos. 
Hay un tipo de trabajos que en sus temáticas plantean problemas desarrollados en los marcos teóricos y metodológicos de otras disciplinas del conocimiento como son: trabajos de historia de la contabilidad y la contaduría pública, de educación o pedagogía, de economía y contabilidad, así como de sociología.

La clasificación de los enfoques de investigación se realiza en el ámbito de la disciplina contable, sin embargo, los miembros académicos del Ccinco tienen trabajos en el ámbito profesional, político gremial, histórico y educativo.

Los primeros artículos publicados por Jack Araujo y Rafael Franco Ruiz plantean problemáticas político-gremiales de la profesión, producidos en un contexto caracterizado por la lucha gremial de los contadores por el mercado laboral, a raíz de la llegada de las multinacionales de auditoría a Colombia.

Después de reglamentar la profesión en 1960 y elevar en 1962 la contaduría pública a nivel universitario se abordan, en las publicaciones analizadas, las problemáticas sobre la formación de los contadores, la historia de la enseñanza contable, la educación y desarrollo, la definición del perfil del contador público, entre otros.

Al finalizar la década del ochenta del siglo pasado, Rafael Franco socializa sus primeros trabajos sobre revisoría fiscal, en los que evalúa el proceso de su reglamentación y propone la articulación de esta actividad con su modelo de contabilidad integral. La participación de Franco en la defensa de la revisoría fiscal en Colombia ha sido permanente durante su trayectoria académica.

Una vez reconocida la importancia de la investigación contable para el desarrollo de la contabilidad, por parte del gremio contable, así como la creación del Ccinco, se socializan, en los congresos colombianos de contadores públicos, propuestas que vinculan la investigación contable con los aspectos académicos-gremiales que posibilitan la unidad del gremio.

Por otra parte, durante la década de los noventa del siglo veinte se presentaron trabajos relativos a la historia de la contaduría pública, el control, la revisoría fiscal y la auditoría. Lo que ha caracterizado a las publicaciones de los primeros años del siglo veintiuno ha sido la publicación de textos sobre educación contable, ejemplo de ello es el libro del Hacer al saber: realidades y perspectivas de la educación contable en Colombia, que reúne los esfuerzos de cinco miembros académicos del Ccinco. 
Después de analizar los textos disciplinares producidos en el Ccinco, surgen diversos enfoques de investigación en los que se presentan sus características generales, los aportes de los trabajos adscritos a cada uno y un cuadro resumen, en el cual se expone la hipótesis central de cada enfoque.

\section{Enfoque del comportamiento desde una perspectiva sociológica}

En este enfoque se reconoce el carácter social de la disciplina contable, su incidencia en la configuración y sostenimiento de las estructuras sociales y su impacto en las transformaciones de la misma.

Una de las hipótesis adscrita a este enfoque es la que plantea Danilo Ariza en la que señala lo siguiente: "La contabilidad ha sido básicamente la expresión de una relación social cuya esencia radica en una racionalidad controladora de la riqueza social que se produce y adscribe a una propiedad" (2002: 71). Este supuesto ha sido trabajado por el autor desde distintos aspectos.

En primer lugar, en su ponencia "Una aproximación a la naturaleza de la contabilidad" esboza la hipótesis tomando como referente la teoría marxista: "El patrimonio es consecuencia de la utilidad, es la forma social que ha ido tomando la apropiación de la plusvalía, según las formas de producción que se han ido sucediendo" (1996: 13). En ese momento la hipótesis no se presenta de manera precisa.

En "Sociedad, conocimiento y contabilidad" (1990-91: 10) Ariza señala: "la contabilidad es una clase de conocimiento circunscrito a la esfera de lo social, consistente en captar e informar en forma objetiva lo relacionado con la existencia, transformación y acumulación del patrimonio".

Luego en su trabajo "Una aproximación dialéctica de la contabilidad" (1990: 50) Ariza plantea que "el objeto de estudio de la contabilidad es la existencia de transformaciones de la riqueza adscrita a una propiedad", señalando la importancia de aprehender el objeto de estudio de la contabilidad para elaborar su conocimiento científico.

\footnotetext{
${ }^{3}$ Esta ponencia fue escrita en 1984 y publicada por el autor en diciembre de 1996 en la revista Lúmina No. 1 de la Universidad de Manizales.
} 
Dos años más tarde, en su texto "La contabilidad: objetivación del control económico-social" (1992: 5) indica: "la contabilidad es el tipo de información y comunicación que tiene como objeto de estudio el control social económico en torno a la riqueza adscrita a una propiedad". Con esta afirmación Ariza retoma la definición de Jack Araujo acerca de la contabilidad como un sistema de información y comunicación.

En el ámbito disciplinar, este autor estudia a la contabilidad en el ámbito del medioambiente manteniendo su hipótesis de que "La contabilidad ha sido básicamente la expresión de una relación social cuya esencia radica en una racionalidad controladora de la riqueza social que se produce y adscribe a una propiedad" (2002: 71), con lo cual abre nuevas posibilidades de explicación para el desarrollo de la teoría contable, así como señala la necesidad de reconceptualizar el ente económico con el fin de internalizar sus responsabilidades en el contexto natural y social en el que actúa, de cuya preservación depende su existencia.

Ariza en su texto "La inserción contable en la contradicción del desarrollo sostenible" (2000a': 2-4) señala: "la contabilidad es percibida como la racionalidad controladora que en cada modo de producción identifica la coherencia que debe vincular la dinámica de la producción con la dinámica de la distribución o apropiación" y continúa "la contabilidad como práctica social está constituida por procedimientos, conceptos y normatividades, expresables de símbolos que inventan y crean las formas de comprender".

En "Una perspectiva para captar la inserción contable en la problemática medio ambiental" (2000b: 582) retoma planteamientos de su artículo anterior y manifiesta que "la contabilidad percibida como la racionalidad controladora, que coadyuva a la reproducción de la entidad productora, ha hecho énfasis en el vínculo de las dinámicas de producción, distribución (apropiación) predominantes en cada uno de los modos de producción". En este texto mantiene la hipótesis presentada al inicio y describe a la contabilidad como una racionalidad controladora insertándola en el discurso del medioambiente desde una perspectiva de responsabilidad social, así como estableciendo una equidad en la distribución social de la riqueza.

En el artículo "La interdisciplinariedad contable como clave de su supervivencia social" (2002:71) expresa lo siguiente: "La contabilidad ha sido básicamente la expresión de una relación social cuya esencia radica en una racionalidad controladora de la riqueza social que se produce y adscribe a una propiedad". En esta 
hipótesis existen cambios conceptuales con relación a las anteriores. En sus dos primeros textos Ariza indica que la contabilidad incluye e informa la existencia, transformación y acumulación del patrimonio ${ }^{4}$. Por otro lado, en sus dos artículos, publicados en los primeros años de la década de los noventa del siglo pasado analiza el control socioeconómico como objeto de estudio de la contabilidad; posteriormente, en sus publicaciones de 2000 a 2002, señala a la racionalidad controladora de la riqueza como la esencia ${ }^{5}$ de la contabilidad.

En su trabajo "La contabilidad objetivación del control económico-social a través de los diversos modos de producción" (1992) Ariza percibe a la contabilidad como una disciplina social a partir de la problemática y dinámica de una de las funciones básicas del hombre, la producción. Cuando incluye a la contabilidad en la problemática medio ambiental hace énfasis tanto en la producción como en la distribución (apropiación) de la riqueza.

\section{Cuadro 1}

Resumen del enfoque del comportamiento desde una perspectiva sociológica

\begin{tabular}{cccc}
\hline Año & Autor & Título del trabajo & Hipótesis central \\
\hline 2002 & Danilo Ariza & La interdisciplinariedad & La contabilidad ha sido básicamente la expresión \\
& contable como clave de & se una "relación social" cuya esencia radica en una \\
& & & $\begin{array}{c}\text { racionalidad controladora de la riqueza social que se } \\
\text { produce y adscribe a una propiedad. }\end{array}$ \\
\hline
\end{tabular}

Fuente: Elaboración propia

\section{Enfoque normativo deductivo}

A partir de este enfoque normativo deductivo se definen las normas para la práctica contable que tienden a buscar la verdad y que se plasman en prescripciones normativas; desde esas teorías valorativas, se observa y mide la realidad contable en la que sus planteamientos son a priori a la observación (planteados desde el deber ser).

\footnotetext{
${ }^{4}$ A partir de 1990 Ariza cambia el concepto de patrimonio por el de riqueza adscrita a una propiedad, aunque sus explicaciones con relación a este concepto se presentan desde sus primeros trabajos.

${ }^{5}$ Los estudios que realiza este autor sobre la esencia se encuentran en su trabajo "Una aproximación dialéctica de la contabilidad" en 1990.
} 
En los aportes a este enfoque se encuentran los trabajos disciplinares de Édgar Gracia, en su mayoría artículos y ponencias, a través de los cuales es evidente su propuesta de fundamentación de la contabilidad desde marcos teóricos propios de la economía. Sus hipótesis están adscritas al enfoque deductivo normativo, pues buscan la solución de problemas fundamentales de la contabilidad en conceptos económicos de valor, renta y riqueza para la construcción de teorías valorativas. Este planteamiento está presente en su obra, en la cual es evidente cómo se van transformando sus hipótesis de trabajo.

Este mismo autor en "Contabilidad y realidad socioeconómica - Consideraciones para la construcción del sistema de valores" (1991) menciona que "la contabilidad asume el criterio de medir básicamente el ingreso monetario; reduce el esquema de cuentas desconociendo la noción de riqueza o mejor [sic] apropiándose de una concepción donde el factor trabajo no forma parte (aparente) de la estructura de medición [...]"(1991:175) y concluye "La actual contabilidad no es sistémica, al no involucrar la concepción de tiempo social de valorización de la riqueza" (1991:176).

Seis años después en su trabajo "Elementos metodológicos para la construcción del control económico ambiental" (1997) Gracia manifiesta que "El principal problema que enfrenta la estructura del control económico ambiental tiene que ver con el abordaje y clasificación de los sistemas e instrumentos de medición empleados; su resolución permitirá establecer no sólo parámetros de valoración, sino también factores de clasificación y ordenación de los recursos económicos y ambientales" (1997:35).

Esta hipótesis la concreta en el trabajo "Economía y sistema de cuentas" (2000a), donde presenta la siguiente afirmación con pretensión explicativa: "los sistemas de cuentas y balances, los modelos de contabilidad nacional y de empresa, en su construcción, se descubren como resultado de una concepción positivista que basa sus descripciones en observaciones particulares de los hechos y fenómenos, fragmentando la realidad descrita, situación que deriva en la presencia de estructuras contables aisladas con objetivos diversos y no congruentes, convertidas en simples formalidades que no ofrecen soluciones efectivas en materia de decisiones y articulaciones con los planes generales, sectoriales y empresariales de la economía social" (2000a:18). 
En el trabajo "El contexto de la contabilidad de productividad" (2002) Gracia retoma la hipótesis presentada en sus trabajos: "La contabilidad — como instrumento de planificación y decisión — ha resultado imbuida de la racionalidad práctica estratégica que proyectan las organizaciones para enfrentar la competencia. Esto es así si se tiene en cuenta que la competitividad se desarrolla a partir de una racionalidad práctica que prospera a través de una lógica instrumental, que se ha convertido en estratégica en tanto utiliza como medio para alcanzar sus fines, las expectativas recíprocas de los sujetos. En este sentido, la contabilidad se convierte en un instrumento estratégico, de predicción del comportamiento y de las expectativas de los sujetos, antes que responder al simple registro de las operaciones pasadas de la empresa" (2002: 63).

Estos trabajos de Gracia son aproximaciones al estudio de la contabilidad desde marcos teóricos de la economía; hasta el momento dichos planteamientos no han sido contrastados empíricamente.

Hay otros trabajos como "Importancia del desarrollo tecnológico de la contabilidad" (Gracia, 1992), "El control económico en el marco de la producción, distribución y consumo" (Gracia, 1995) y "A propósito del contexto económico y los escenarios" (Gracia, 1996), en los cuales se aborda la tecnología como instrumento de construcción científica de la contabilidad, la importancia del control económico para el estudio de la contabilidad y una mirada al contexto económico y al paradigma científico tecnológico y su relación con la construcción de la contabilidad como disciplina. Estos textos constituyen la evidencia del interés de Gracia en el contexto de la contabilidad, estableciendo desde otras esferas posibles problemas de investigación y define la economía como el referente o marco teórico a partir del cual se puede comprender el campo de estudio de la contabilidad.

La propuesta de Harold Álvarez y María Dolly García González sobre una "Formulación metodológica para las cuentas regionales" se refiere a este enfoque; en él los autores afirman: "que es posible la obtención de los datos base de la matriz insumo/producto para un sector de la economía a partir de los datos de las contabilidades de las unidades microeconómicas, mediante un proceso de consolidación contable" (2001: 105).

Los autores retoman consideraciones de la economía, trabajando como marco referencial los enfoques matricial y conjuntista, para representar la consolidación de la macrocuenta, tomando el teorema de consolidación propuesto por Mattessich, 
en el cual — según los autores - se demuestra que "dos o más balances de prueba $B_{l}^{s}, \ldots, B_{m}^{s}$ de entidades diferentes $e_{l}, \ldots, e_{m}$ al final de un periodo t, se pueden consolidar en un solo balance $B_{n}^{s}$ de una entidad $e_{n}^{n}$ " (Álvarez y García, 2001:16). Este teorema, la matriz insumo producto y la información contable planifican la producción y el consumo de los entes.

Asimismo, una parte de las hipótesis de Hernán Quintero también pueden adscribirse al enfoque normativo deductivo, pues incluye en estas categorías conceptuales al capital y la riqueza. Las otras hipótesis expuestas corresponden a reflexiones contables desde la epistemología.

En su última publicación "Perspectivas de la investigación contable" Quintero afirma: "La contabilidad es un saber estratégico sustentado por la razón práctica para ponerlo al servicio del capital" (2002:122). En esta hipótesis de trabajo retoma planteamientos de Carlos Ortiz (1988) de la contabilidad como saber estratégico. También señala: "la categoría riqueza es el referente orientador de la investigación contable para dar cuenta de cómo se produce y distribuye socialmente" (2002:63).

En su trabajo disciplinar: "Hacia una escuela de investigación contable en Colombia" Quintero dice que "La contabilidad como fuerza importante para el control de la economía es milenaria y convertida en saber estratégico se enruta (sic) en la defensa de la propiedad privada" (1996: 22); y añade "La contabilidad es un fenómeno universal del conocimiento de alta complejidad que hace parte de los procesos económicos, sociales y semióticos" (1996:25). También retoma la definición de Carlos Miguel Ortiz: "la contabilidad, entendida y explicada como sistema de información y comunicación de valores de cambio dentro de una organización, en un momento determinado y desde su dimensión cuantitativa" a lo que Quintero agrega que también es cualitativa. Por último, plantea "la contabilidad es un objeto de conocimiento multiparadigmático"(1996:25).

Cinco años más tarde, en su trabajo "La lógica de la investigación contable", Quintero 1995) niega la existencia de los objetos de estudio y las disciplinas, tomando como referente a Popper (Conjeturas y refutaciones); por ello, afirma: "Los objetos de estudio de la contabilidad son múltiples y desbordan las fronteras de los demás saberes [...] No estudiamos temas, sino problemas y los problemas pueden atravesar cualquier objeto de estudio o disciplina" (1995: 18). En este trabajo Quintero presenta una elaboración sobre semiótica y contabilidad, por lo que señala: "los 
estados financieros son estructuras simbólicas constituidas a través del tiempo" (1995: 23). Esta hipótesis no tiene continuidad en sus trabajos posteriores.

Posteriormente, en su trabajo "La contabilidad en el umbral de la modernidad", Quintero (1997) incursiona en la problemática del medioambiente (aunque este trabajo no tiene continuidad); en este mismo texto desarrolla más su hipótesis de la contabilidad como saber estratégico al servicio del capital, la cual presenta en el trabajo ya mencionado "Perspectivas de la investigación contable en Colombia".

\section{Cuadro 2}

\section{Resumen del enfoque normativo deductivo}

\begin{tabular}{clll}
\hline Año & \multicolumn{1}{c}{ Autor } & \multicolumn{1}{c}{ Título del trabajo } & \multicolumn{1}{c}{ Hipótesis central } \\
\hline $2002-3$ & $\begin{array}{l}\text { Èdgar Gracia } \\
\text { López }\end{array}$ & $\begin{array}{l}\text { El contexto de la } \\
\text { contabilidad de } \\
\text { productividad. }\end{array}$ & $\begin{array}{l}\text { La contabilidad -como instrumento de planificación y decisión- } \\
\text { ha resultado imbuida de la racionalidad práctica estratégica que } \\
\text { proyectan las organizaciones para enfrentar la competencia. }\end{array}$ \\
2001 & $\begin{array}{l}\text { Harold } \\
\text { Álvarez y } \\
\text { María Dolly } \\
\text { García }\end{array}$ & $\begin{array}{l}\text { Formulación } \\
\text { metodológica para las } \\
\text { cuentas regionales. }\end{array}$ & $\begin{array}{l}\text { Es posible la obtención de los datos base de la matriz Insumo/ } \\
\text { Producto para un sector de la economía a partir de los datos de } \\
\text { las contabilidades de las unidades microeconómicas, mediante } \\
\text { un proceso de consolidación contable. }\end{array}$ \\
& $\begin{array}{l}\text { Hernán } \\
\text { Quintero }\end{array}$ & $\begin{array}{l}\text { Perspectivas de } \\
\text { la investigación } \\
\text { contable. }\end{array}$ & $\begin{array}{l}\text { La contabilidad es un saber estratégico sustentado por la razón } \\
\text { práctica para ponerlo al servicio del capital. }\end{array}$ \\
\hline
\end{tabular}

Fuente: Elaboración propia

\section{Enfoque de los modelos de decisión y la capacidad predictiva}

En este enfoque se identifica la información más relevante en función de su potencial con respecto al suministro de datos predictivos; igualmente, se estudia la utilidad de las cifras contables.

La obra académica de Jack Araujo aporta nuevos elementos a este enfoque al identificar la contabilidad como información que produce mensajes para la toma de decisiones y conocimiento de la realidad y situación económica de las organizaciones; esta idea, desarrollada en su trayectoria académica, incluye al enfoque de los modelos de decisión y capacidad predictiva porque identifica la utilidad y la relevancia con la capacidad de la información para predecir hechos futuros y para informar sobre la situación económica, política y social de las organizaciones. 
Este enfoque de Araujo se puede observar en "Elementos para una investigación en contabilidad" (1979:7-8) cuando plantea los problemas de la contaduría pública como primarios — que forman parte de la lucha gremial por la defensa del mercado de trabajo de los contadores colombianos ante las multinacionales- y secundarios que tienen que ver con las deficiencias de las técnicas que producen la información (el modelo de normas es implantado de otra realidad) y lo referente a lo académico, dentro de lo cual ubica a la investigación en contabilidad. A partir de este trabajo, Araujo delimita los problemas de la contabilidad, que estudia desde 1979 , e identifica los elementos que se deben tener en cuenta para realizar investigación científica en contabilidad: 1) Una clara y correcta definición de contabilidad; 2) crítica de las bases ideológicas en las cuales se encuentra en la actualidad la contabilidad; 3 ) establecer claramente su estructura; 4) no tomar como punto de partida el interés que pueden tener determinados sectores económicos, ni siquiera el sistema en su conjunto; 5) tomar como punto de partida su objeto mismo como sistema informativo; y 6) hacer abstracción del valor dinero como forma de medida y verla, en cambio, en su forma más natural y original.

Las preguntas planteadas se encuentran en el artículo con que inicia su trayectoria en la construcción de la contabilidad de manera disciplinar. A continuación se contrasta con las hipótesis trabajadas en sus textos.

Después de analizar las definiciones existentes en contabilidad menciona: "Si entendemos a la contabilidad como un sistema informativo y de comunicación de hechos económicos, debemos entender que su estructura está formada por cuatro elementos: informador (contador), medios informativos (los libros, los computadoras, etc.), el mensaje (informes y estados financieros) y los receptores (los usuarios)". Esta hipótesis la desarrolla en el texto "Un nuevo concepto de contabilidad" (1981:2).

Un año más tarde, en "El método científico en el estudio de la evolución del pensamiento contable", Araujo plantea: "Lo esencial en la contabilidad no es la partida doble, sólo es importante para el mensaje financiero, pero no para el resto de mensajes que la contabilidad puede producir. El mensaje de la contabilidad es múltiple y se ha ido especializando: en un principio existió la partida simple; luego, con el surgimiento del comercio, la partida doble con un mensaje de tipo comercial y financiero; posteriormente, con la aparición de la industria, tenemos un mensaje relativo a los costos de producción; con la sociedad anónima y la intervención del Estado en la empresa, surge el mensaje hacia el público, hacia la sociedad. De más reciente aparición tenemos los mensajes administrativos, el social, relativo al re- 
curso humano de la empresa; el nacional, referente a la actividad macroeconómica del país, etc." (1983:25).

La segunda hipótesis del artículo "Elementos para la investigación" se desarrolla y precisa en el trabajo "Por una epistemología para la contabilidad"; en él Araujo afirma: "El conocimiento contable no es objetivo, ya que sus enunciados básicos (axiomas) no concuerdan aproximadamente con la realidad u objeto sobre el cual trabaja; además, no es verificable en la mayoría de los casos ya que no existe forma de confirmarlo con los hechos mismos, sino que se aceptan como correctos o válidos en la medida en que han sido referenciados con un conjunto primario de ideas básicas sin correspondencia en los hechos mismos" (1987a:9).

Por su parte, en el artículo "Una nueva ecuación para la contabilidad A=P+C+O" este mismos autor mantiene su hipótesis alrededor de la contabilidad como comunicadora de un mensaje: "Hoy en día las empresas han crecido tanto que la ecuación contable resulta insuficiente para comunicar todas las operaciones que realizan y los recursos de que disponen. Debemos agregar a la ecuación la letra $\mathrm{O}$, que significa: otras fuentes de financiamiento, y así la tradicional ecuación contable quedaría: $\mathrm{A}=\mathrm{P}+\mathrm{C}+\mathrm{O}$. De esta forma todos los activos que se adicionan en a la ecuación del lado de la izquierda A, tendrán su correspondiente incremento del lado derecho con la letra $\mathrm{O}$. Así ya no será restrictiva la ecuación apara comunicar su real situación financiera" (1987b:5-7).

En "El concepto de utilidad" Araujo evidencia su interés por la contabilidad social e identifica que su finalidad: "Medir la satisfacción de las necesidades sociales por parte de la empresa, tanto en el interior como en el exterior de la misma, y en la medida que pretenda calcular la satisfacción de las necesidades, está calculando la utilidad que produce y aplica en esa gestión social" (1987c:10).

Los trabajos disciplinares de Jack Araujo abordan falencias de las normas al generalizar procedimientos alrededor de los hechos económicos y las hipótesis planteadas son instrumentales, orientadas hacia la resolución de problemas técnicos contables. Aunque en su obra menciona problemas metateóricos y teóricos de la disciplina, no son desarrollados.

Después de 1988, el autor desarrolla otros trabajos disciplinares alrededor de las críticas a la reglamentación de la contabilidad y a la contabilidad social, centrándose en su interés sobre la dimensión social y ambiental de la contabilidad. Esta 
concepción fue evidenciada en uno de los textos mencionados anteriormente que constituye la base de su trabajo académico: "[...] hay enfoques que establecen que la contabilidad es una disciplina que no tiene por qué fundamentar todo su proceso informativo sobre la base económica, sino que también puede informar hechos sociales, políticos o de cualquier otro orden"(1987c:10).

La segunda parte de la obra de Araujo describe temas relacionados con la contabilidad y específicamente de la contabilidad social. Éstas son las principales afirmaciones de sus publicaciones:

- Balance social. Su análisis e interpretación (Araujo, 1989:108). Es un texto descriptivo que presenta definiciones sobre la estructura de los informes y balances sociales. En él es posible observar la permanencia de la concepción de la contabilidad como un sistema informativo y de comunicación social de hechos económicos, financieros, administrativos, sociales, etcétera.

- Ciencia y tecnologías contables (o la verdad en contabilidad). Este texto se relaciona con las concepciones del autor, mantenidas a lo largo de su obra, "[...] de los cuatro elementos constitutivos de la contabilidad, uno de ellos, los medios, está ligado al desarrollo tecnológico. Los tres restantes: el comunicador, el receptor y el mensaje, están relacionados con los avances científicos en contabilidad" (Araujo, 1993a:3).

- Un nuevo paradigma contable para Colombia: La utilidad de la información -o el Decreto 2649 de 1993. "La misión de la contabilidad es proporcionar información tanto a las personas naturales y jurídicas, como a la sociedad en general, para satisfacer sus necesidades de ésta en sus decisiones por tomar, así como en los procesos de mediciones de los cambios, control y evaluación de la producción, distribución y consumo de los recursos" (Araujo, 1993b:133).

- Qué es y qué no es contabilidad. Este texto fue publicado en 1997 (escrito en 1981), y corresponde a la etapa en la cual persiste en su interés por definir contabilidad: "La contabilidad es un sistema informativo y de comunicación de hechos económicos y sociales, integrado por cuatro elementos: el comunicador (contador) los medios de comunicación (libros, máquinas, etc.), el mensaje (estados o informes, etc.) y los usuarios (administración, accionistas, etc.)" (Araujo, 1997:103). 
Las afirmaciones centrales de los textos de Jack Araujo implican una pretensión explicativa de qué es la contabilidad sin configurar aún categorías conceptuales para constituir una hipótesis.

\section{Cuadro 3}

\section{Resumen del enfoque de los modelos de decisión y la capacidad predictiva}

\begin{tabular}{cccc}
\hline Año & Autor & $\begin{array}{c}\text { Título del } \\
\text { trabajo }\end{array}$ & Hipótesis central \\
\hline 1997 & Jack & Qué es y qué no & La contabilidad es un sistema informativo y de comunicación de hechos \\
& Araujo & es contabilidad & $\begin{array}{c}\text { económicos y sociales integrado por cuatro elementos: el comunicador } \\
\text { (contador) los medios de comunicación (libros, máquinas, etc.) el mensaje } \\
\text { (estados o informes, etc.) y los usuarios (administración, accionistas, etc.). }\end{array}$ \\
\hline
\end{tabular}

Fuente: Elaboración propia

\section{Enfoque integrado: neopositivismo inductivo}

En este enfoque se estudian las relaciones que unen a los diversos grupos de usuarios y el papel que en ellas desempeña la información contable.; asimismo, aborda la disciplina contable desde una posición empírica; utiliza líneas de investigación inductivas con hipótesis basadas en las teorías de la agencia y la red contractual; además, analiza la incidencia de las cifras contables en el comportamiento y decisiones de la gerencia, en la hipótesis de la red contractual que correlaciona la participación (representada en cifras contables) de los diferentes estamentos en una entidad y la hipótesis de la eficiencia que analiza los efectos ocurridos en la empresa a causa de los cambios contables. La investigación empírica "Políticas y normas contables en una muestra de Sociedades Anónimas del Valle del Cauca" (1990) de Jorge Burbano es un ejemplo de este enfoque.

Desde marcos de referencia de la teoría de la agencia, tomados del libro Positive Accounting Theory de Watts y Zimmerman ${ }^{6}$, Jorge Burbano valida algunas hipótesis sobre dicha teoría, para ello revisa las prácticas contables existentes en las 27 empresas seleccionadas como muestra; además, recoge información sobre las políticas y normas utilizadas en éstas.

\footnotetext{
${ }^{6}$ Los fundadores del enfoque del neopositivismo inductivo presentan sus primeras aportaciones en 1979.
} 
Desde sus primeros trabajos, Jorge Burbano trabajó la hipótesis: El sistema de información contable de la organización es un sistema formal, de información y comunicación de hechos económicos y sociales.

En su artículo "Unidad de análisis para el estudio del sistema de información contable" Burbano señala que "La contabilidad es el mayor sistema de información económica financiera que existe en las empresas" (1985:6)

Cinco años más tarde, en su texto "Políticas y normas contables en una muestra de sociedades anónimas del Valle del Cauca" (señalado anteriormente) afirma que "La contabilidad concebida como sistema de información es una herramienta importante de la administración y los encargados de elaborar los informes" (1990:89).

En su ponencia "Las normas contables en Colombia: una aproximación histórica crítica y alternativas de solución" indica: "[..."] la contabilidad es una herramienta al servicio de la administración" (1991:30). En este trabajo incluye los resultados de su investigación empírica anterior para soportar la reflexión histórica que realiza sobre las normas contables en Colombia.

Más tarde Burbano en su texto "Reflexión sobre enfoques contables normativo y positivo" plantea que "La contabilidad es una tecnología que alcanza su estatus si al menos está compuesta por conocimientos teóricos y aplicaciones dentro de una estructura social dada" (1993:10).

En sus hipótesis define la contabilidad como proceso, sistema de información o instrumento de información; la mayoría de sus trabajos tiene referente empírico, dada su concepción de la contabilidad como tecnología que se sustenta en la teoría y la práctica.

\section{Cuadro 4}

Resumen del enfoque integrado: neopositivismo inductivo

\begin{tabular}{|c|c|c|c|}
\hline Año & Autor & Título del trabajo & Hipótesis central \\
\hline 1985 & $\begin{array}{c}\text { Jorge } \\
\text { Burbano }\end{array}$ & $\begin{array}{l}\text { Políticas y normas contables en una } \\
\text { muestra de sociedades anónimas del } \\
\text { Valle del Cauca }\end{array}$ & $\begin{array}{l}\text { El sistema de información contable de la } \\
\text { organización es un sistema formal, de información } \\
\text { y comunicación de hechos económicos y sociales. }\end{array}$ \\
\hline
\end{tabular}

Fuente: Elaboración propia 
Textos no adscritos a los enfoques de investigación propuestos

Algunos textos presentan rasgos insuficientes para incluirlos en los enfoques de investigación propuestos, dado el tipo de argumentación que presentan; no son argumentos deductivos — explicativos- en los que las conclusiones formales (lógicamente) se puedan derivar de las premisas, implícitamente y para que el argumento deductivo sea válido formalmente, pues se supone que las premisas son suficientes y que lo que se hace al concluir es hacer explícita la explicación. De la misma manera, otros textos presentan argumentos inductivos, que proporcionan información nueva soportada en las premisas en las que su validez consiste en que sea posible una conclusión, dado la verdad de las premisas. ${ }^{7}$

Las temáticas propuestas por Rafael Franco, a través de su producción académica, abordan diversos ámbitos: profesional, político-gremial, educativo y disciplinar (a nivel metateórico, teórico y técnico). Los textos analizados para su clasificación en enfoques de investigación corresponden sólo al ámbito disciplinar, en los cuales plantea su propuesta permanente de contabilidad integral y realiza reflexiones sobre temas epistemológicos. La producción académica de Franco presenta afirmaciones generales con las cuales es difícil ubicarlas en el enfoque de investigación propuestos.

En el libro Reflexiones contables (1984-5) Franco incluye ponencias de congresos y encuentros de contadores, aunque algunos trabajos los presenta inconclusos.

Posteriormente, en Contabilidad integral: teoría y normalización (1995) presenta su propuesta de un modelo de contabilidad integral y señala que "existe una nueva forma de concebir la contabilidad, fundamentada por una metodología tecnológica y un enfoque económico de planeación, para mostrar una dimensión más profunda y rigurosa de procedimientos contamétricos soportados sobre conceptos teóricos universales que se construyen en el nivel abstracto de la teoría contable" (1995:185). En el libro expone afirmaciones generales, definiciones instrumentales sobre los componentes de su modelo, pero no presenta conclusiones sobre su trabajo.

\footnotetext{
${ }^{7}$ Estos conceptos se trabajaron del capítulo II. “Argumentos deductivos y argumentos inductivos” de José A. Díez y Ulises Moulines del libro Fundamentos de filosofía de la ciencia”. Los textos presentan afirmaciones generales, descriptivas, lo que dificulta la constitución de hipótesis de trabajo explicativas.
} 
En su libro Ajustes integrales por inflación (1997) Franco plantea la problemática de la inflación y sus implicaciones tanto en la información contable y tributaria. Presenta las implicaciones de la inflación en lo contable con descripciones generales que no explican las causas del fenómeno. En este trabajo Franco define a la contabilidad "como un conjunto de conocimientos y procedimientos (teoría contable más contametría), acerca de hechos y fenómenos de la actividad socioeconómica" (1997:75); además, presenta una propuesta para el manejo de la información contable en entornos de precios cambiantes, en la que intentan plantear un modelo y un instrumento técnico de operacionalización.

Cabe aclarar que los últimos trabajos de Rafael Franco están inscritos en la temática de historia del pensamiento contable.

Por otra parte, William Rojas en su texto "Intramuros: la contabilidad y la organización" aborda las problemáticas que existen en el ámbito disciplinar; del mismo modo presenta varias propuestas sobre la contabilidad a la que señala como "la disciplina del conocimiento que agrupa las teorías, conceptos y técnicas, en virtud de las cuales se procesan, analizan e interpretan los hechos económicos y sociales para la planificación, el control y la toma de decisiones en las organizaciones" (1999:104).

Más tarde Rojas menciona que "la contabilidad es una práctica social con inquietudes "científicas" y con un sentido notoriamente público, ligado a la historia de las organizaciones" (1999:113) y, finalmente, la señala como un campo de conocimiento. Dado la pluralidad de las definiciones de la contabilidad es compleja ubicarla en uno de los enfoques (disciplinares) propuestos. Se erige en una reflexión que - como señala el autor - pretende de forma panorámica presentar la importancia de la contabilidad y la contaduría en las organizaciones.

En esta misma perspectiva se encuentran los trabajos de Olver Quijano en su texto "Contexto, contabilidad e investigación: una lectura preliminar" (2001); en él plantea problemáticas acerca de los procesos de formación contable, el desfase de los desarrollos contables con relación a las dinámicas socioempresariales y la precariedad de la investigación. El texto constituye una lectura — desde los marcos interpretativos y referenciales del autor- para comprender estas problemáticas; sus hipótesis de trabajo en el ámbito disciplinar no están inscritos a los enfoques propuestos. 
Por su parte, los trabajos de Guillermo Martínez, clasificados en el ámbito disciplinar, son: "Imperio transnacional: transplante y adopción de normas y modelos contables" (1998 y "El entorno y el seudomorfismo conceptual de la regulación contable" (2000); en ellos presenta una lectura del contexto político-económico de los procesos de estandarización contable.

El último artículo analizado de este autor es: "El estatuto de cientificidad de la contabilidad: un debate inconcluso" (2001) en el que aborda temas epistemológicos sobre el objeto y método en contabilidad; las afirmaciones presentadas en el texto son proposiciones secuenciales con coherencia lógica, pero en las que es difícil abstraer una hipótesis central con pretensiones de explicar la problemática planteada. En el texto Martínez plantea la hipótesis aún sin demostrar: "La estructura simbológica de la contabilidad (lo contable, lo que se deriva de la acción de contar) debe estudiarse dinámicamente, dentro de las circunstancias de tiempo, modo y espacio, que condicionan su gestación, y dentro de una perspectiva de devenir inacabado, falible, siempre abierto a cambios y transformaciones permanentes. Esta afirmación es proposición secuencial que no logra constituirse en una hipótesis.

En los trabajos hay afirmaciones desde referentes políticos y epistemológicos con aportes a la reflexión sobre la contabilidad, aunque sin proponer hipótesis de trabajo disciplinares, por lo cual no es posible adscribirlos a algunos de los enfoques propuestos.

\section{Conclusión}

Las problemáticas planteadas en los primeros años de desarrollo de los procesos de investigación contable se centran en reflexiones sobre temas epistemológicos de la contabilidad: la aproximación a su naturaleza, su objeto y método de estudio. Estas reflexiones se abordan desde marcos referenciales de la filosofía de la ciencia y la sociología, aproximándose a la contabilidad, sin lograr su problematización, pues se permanece en los marcos que se proponen para su interpretación.

Posterior a la expedición del Decreto 2160 de 1986, se concentran las críticas a la normatividad contable, solicitándose la derogación del mismo. En las críticas se señalan las implicaciones del fenómeno inflacionario en la información contable y la necesidad de contextualizar la norma. Cuando se expide el Decreto 2649 de 1993 se recoge gran parte de las discusiones académicas planteadas entre 1986 y 1993. 
Cuando se inician los simposios de investigación contable y docencia del Ccinco, en 1990 se presentan nuevas reflexiones epistemológicas de la contabilidad. Se realizan aproximaciones a problemas de la contabilidad: medición de la riqueza, control económico-ambiental, control económico-social, valoración ambiental, representación contable, entre otros, utilizándose para su planteamiento marcos referenciales de otras disciplinas: economía, sociología y lingüística.

La producción académica, presentada en los primeros años del siglo XXI por los miembros académicos del Ccinco en el ámbito disciplinar, tiene la característica de abordar de manera tangencial problemas de la disciplina contable desde los marcos interpretativos y referenciales de sus nuevos miembros, ampliando las perspectivas del desarrollo de la disciplina, fortaleciendo la comprensión de las problemáticas planteadas, pero distanciándose de los problemas teóricos de la contabilidad.

En los primeros trabajos abordados veíamos que los autores asumían la contabilidad como ciencia, adjudicándole objetos y métodos de estudio. Posteriormente, al aproximarse a su problematización la reconocen como una disciplina científica o disciplina del conocimiento en construcción, aún sin profundizar en su caracterización como disciplina.

\section{Referencias}

Álvarez, H. (2001). Formulación metodológica para las cuentas regionales. Armenia: Universidad del Quindío.

Araujo, E. J. (1979). Elementos para una investigación en contabilidad. Medellín: Adeconti.

(1981). "Un nuevo concepto de contabilidad. Teoría J", ponencia presentada en el III Congreso de Contadores del Pacto Andino, Bogotá.

(1983). "El método científico en el estudio de la evolución del pensamiento contable", Investigación contable, 1, 16-29.

(1987a). "Por una epistemología para la contabilidad", Investigación Contable, 5-6, 4-21. 
(1987b). "Una nueva ecuación para la contabilidad: A = P + C + O", Investigación Contable, 3, 4-17.

(1987c). "El concepto de utilidad2, Investigación Contable, 4, 4-11.

(1989) “El balance social. Su análisis e interpretación”, Contaduría, 15.

(1993a). "Ciencia y tecnologías contables (o la verdad en contabilidad)", Colegio Colombiano de Contadores Públicos, 13.

(1993b). "Un nuevo paradigma contable para Colombia: La utilidad de la información —o el Decreto 2649 de 1993-“", Contaduría, 24-25, 127-137.

(1996). "Qué es y qué no es contabilidad". Lumina, 2, 101-104.

ARIZA, B. D. (1990). "Una aproximación dialéctica de la contabilidad”, I Simposio de Investigación Contable y Docencia, Medellín.

(1990-1991). Sociedad, conocimiento y contabilidad. Bogotá: Colegio Colombiano de Contadores, 9-10.

(1992). La contabilidad objetivación del control económico-socia,. II Simposio Nacional sobre Investigación Contable y Docencia, Armenia.

(1996). "Una aproximación a la naturaleza de la contabilidad", Lúmina, 1, 4-16.

(2000a). La inserción contable en la contradicción del desarrollo sostenible. Trabajo presentado en V Simposio de Investigación Contable y Docencia, Medellín.

(2000b). "Una perspectiva para captar la inserción contable en la problemática medio ambiental”, Legis del Contador, 4, 161-191.

(2002). "La interdisciplinariedad contable como clave de supervivencia social”, Cuadernos de Administración, 28, 67-86. 
Belkaoui, A. (1993). Accounting Theory, $3^{\mathrm{a}}$ ed., New York: Harcourt Prace Jovanovich College Publishers.

Burbano, J. y D. Delgadillo (1985). "Unidad de análisis para el estudio del sistema de información contable". Cuaderno de administración, 12.

Burbano, J. (1990). "Políticas y normas contables en una muestra de sociedades anónimas del Valle del Cauca", Cuadernos de administración, 17, 62-102.

(1991). "Las normas contables en Colombia: una aproximación histórica crítica y alternativas de solución”, X Congreso de Contadores, Cúcuta.

DíEz, J. y C. Moulines (1997). Fundamentos de filosofía de la ciencia, Barcelona: Ariel.

CCInco (1987). Estatutos de Constitución del Centro Colombiano de Investigación Contable.

Franco, R. R. (1984-5). Reflexiones contables. Teoría, educación y moral, Armenia: Atenas Ltda.

(1995). Contabilidad integral, teoría y normalización, 2ª ed. Armenia: Investigar Editores.

(1997). Ajustes integrales por inflación, $4^{\mathrm{a}}$ ed. Pereira: Investigar.

Gracia, L. E. (1991). “Contabilidad y realidad socioeconómica-consideraciones para la construcción de un sistema de valores". X Congreso Colombiano de Contadores Públicos, Cúcuta.

(1992). "Importancia del desarrollo tecnológico de la contabilidad", II Simposio Nacional sobre Investigación Contable y Docencia, Armenia.

(1995). "El control económico en el marco de la producción, distribución y consumo", XII Congreso de Contadores Públicos, Barranquilla. mina, 1, 53-59. 

del control económico ambiental", XIII Congreso Colombiano de Contadores Públicos, Cartagena.

\section{3-39.}

(2000a). "Economía y sistemas de cuentas", Legis del Contador, 2, (2002). "El contexto de la contabilidad de productividad", Cuadernos de administración, 28, 49-66.

Martínez, P. G. (1998). "Imperio transnacional: transplante y adopción de normas y modelos contables", Porik An, 1, 61-78.

(2000). "El entorno y el seudomorfismo conceptual de la regulación contable", Legis del Contador, 3, 67-84.

(2001). "El estatuto de cientificidad de la contabilidad: un debate inconcluso", Porik An, 5-6, 41-70.

Ortiz, C. (1988). "El contador y la investigación”, Cuadernos de Administración, 14.

QuiJano V. O. (2001). "Contexto, contabilidad e investigación: una lectura preliminar”, Asfacop, 6, 107-130.

Quintero, C. H. (1995). “Lógica de la investigación contable”, III Simposio de Investigación y Docencia, Bucaramanga.

(1996). "Hacia una escuela de investigación científica en la contabilidad”, Lúmina, 1, 22-30.

(1997). "Contabilidad ambiental: en el umbral de la modernidad", Lúmina, 2, 113-116.

(2002). "Perspectivas de la investigación contable", Cuadernos de Administración, 28, 119-136. 
RoJAs, R. W. (1999). “Intramuros: la contabilidad y la organización”, Contaduría, $34,101-117$.

TúA, P. J. (1989). La investigación empírica en contabilidad. La hipótesis de eficiencia". Madrid: Instituto de Contabilidad y Auditoría de Cuentas/ Ministerio de Economía y Hacienda. 